\title{
STRUCTURAL ALTERATIONS OF THE BLADDER INDUCED BY DETRUSOR INSTABILITY. EXPERIMENTAL STUDY IN RABBITS
}

\author{
JOAO L. AMARO, KARINA T. BALASTEGHIN, CARLOS R. PADOVANI, RENATA \\ MONTENEGRO
}

Department of Urology (JLA, KTB, RM) and Department of Statistics (CRP), School of Medicine, State of Sao Paulo University, UNESP, Botucatu, SP, Brazil

\begin{abstract}
Objectives: The aim of this study was to evaluate the histopathological and immunohistochemical alterations induced by detrusor instability in the bladder of rabbits submitted to partial bladder outlet obstruction.

Materials and Methods: Thirty male Norfolk rabbits were divided into 2 groups, a clinical control and a group with detrusor instability. Urine culture, cystometric study, histopathological and immunohistochemical analysis were performed in all animals prior to surgery (M1) and 4 weeks aftersurgery (M2).

Results: Partial obstruction (G2) resulted in a 2.5 fold increment $(\mathrm{p}<0.05)$ in bladder weight when compared to control (G1). Four weeks after surgery, 93\% of animals in G2 developed cystitis. Partial obstruction resulted in detrusor instability at M2 and bladder capacity was significantly increased ( $\mathrm{p}<0.05$ ) from M1 to M2. The incidence of mild to moderate mucosal and adventitious fibrosis at M2 was higher in G2 ( $\mathrm{p}<0.05$ ) when compared to G1. Inflammatory reaction at M2 was statistically higher $(\mathrm{p}<0.05)$ in $\mathrm{G} 2$. There was no difference in muscular hypertrophy between M1 and M2 in G1. However, 67\% of G2 bladders showed a moderate to intense muscular hypertrophy at M2. Hyperplasia of the epithelium was also increased in G2 when M1 and M2 were compared $(\mathrm{p}<0.05)$.

Conclusion: Detrusor instability induced by partial bladder outlet obstruction caused significant histopathological and immunohistochemical alterations in the bladder of rabbits.
\end{abstract}

Key words: bladder; rabbits; bladder outlet obstruction; histopathology

Int Braz J Urol. 2005; 31: 579-86

\section{INTRODUCTION}

Detrusor instability is a common phenomenon in urological practice that may be seen in men with bladder outlet obstruction (BOO) (1) and in women with urge incontinence, causing psychosocial and sexual problems (2).

Experimental model of partial bladder outlet obstruction in pigs (3) and rats (4) showed structural and functional detrusor changes quite similar to those caused by voiding dysfunction in human. In rabbits, morphologic changes often associated with $\mathrm{BOO}$ include progressive denervation and hypertrophy of the bladder wall $(5,6)$.

Physiological changes that occur in the bladder in response to obstruction, which may lead to disorders in function including detrusor instability, are poorly understood. Thus, there is a need for a suitable animal model that mimics the response of the bladder detrusor instability, for further understanding of BOO. 
Our objective was to evaluate the histopathological and immunohistochemical changes in bladder using an experimental model of detrusor instability in rabbits with partial bladder outlet obstruction (PBOO).

\section{MATERIALS AND METHODS}

Thirty-five male Norfolk rabbits weighing between $1.700 \mathrm{~g}$ and $2.820 \mathrm{~g}$ (average $2.140 \pm 200$ ) were randomly divided into 2 groups. Group 1 (G1, n $=15)$ served as clinical control with no surgical intervention. The remaining 20 animals were submitted to laparotomy, and an adjustable polyethylene bracelet was placed around the bladder neck without compression of the urethra, previously catheterized with a $10 \mathrm{~F}$ catheter, as previously described (7). From this group, only 15 rabbits developed detrusor instability verified by cystometric studies one week after surgical intervention. Since the objective of this trial was to investigate vesical alterations in animals with detrusor instability due to partial bladder obstruction, the 5 rabbits that did not show involuntary contraction of the bladder were not included in the trial. Therefore, group 2 (G2) consisted of the 15 animals with detrusor instability.

Urine culture, serum creatinine and cystometric evaluation were performed in all animals at different moments: one week after surgical procedure (M1) and 4 weeks after surgery (M2). Animals with positive urine culture at M1 were treated with 1 $\mathrm{mg} / \mathrm{Kg} /$ day of intramuscular trimethropim.

The cystometric evaluation was performed using the Urobyte ${ }^{\mathrm{TM}} 5000$ computed urodynamics system. To measure intravesical pressure a 10F Foley catheter was used and to measure intraabdominal pressure, a catheter with a rectal balloon was used (8). During this exam, the vigil animals were placed in wooden cages, with posterior extremities held by cotton strings. After antisepsis of the penile area with topic povidone and lubrication with $2 \%$ xylocaine ${ }^{\mathrm{TM}}$ jelly, a 10F double-way Foley catheter was introduced into the bladder with vesical emptying, and urine was drawn in a sterile tube for urine culture.

After lubricating the rectal balloon with $2 \%$ Xylocaine ${ }^{\mathrm{TM}}$ jelly, it was introduced $2 \mathrm{~cm}$ beyond the anal margin and connected to a 2-way tap, injecting $0.4 \mathrm{~mL}$ of sterile water in one of the ways in order to the balloon wall and the rectal mucosa came into contact. The other way connected to the pressure transmitter. The vesical catheter was connected to a 2-way tap, and one of the ways was connected to the pressure transmitter and the other to a continuous infusion pump ( $2 \mathrm{~mL} / \mathrm{min})$. To verify the good placement of the catheters a slight compression of the abdomen was made in order to obtain an abdominal pressure curve, which was transmitted to the bladder, and, consequently obtaining the vesical curve.

The cystometry was initiated proceeding to the simultaneous measurements of the abdominal and vesical pressures. When urinary leakage around the vesical catheter was observed, the exam was discontinued. At that moment, we verified the vesical pressure (VP) and the abdominal pressure, also measured in the beginning of the urinary leakage (VP-AbdP) expressing maximal detrusor pressure (DeiP). Maximal bladder capacity was considered the one, which attained a filling volume enough to yield bladder contractions. Vesical compliance was obtained by the formula: Fv-Iv/Fp-Ip where Fv = Final volume, Iv = Initial volume, $\mathrm{Fp}=$ Final pressure and $\mathrm{Ip}=$ Initial pressure. Uninhibited contractions were considered those detrusor involuntary contraction with low vesical volume, yielding or not simultaneous urinary leakage.

At M2, after the cystometric evaluation, blood and urine collection all animals were euthanised and their bladders collected and weighed using a laboratory micro scale. Samples from the bladder were fixed in formaldehyde for 24 hours. Then they followed the protocol of the automated inclusion processor Leica TP-1020, where they were dehydrated in 100\% ethanol, followed by clarification in xylol. The samples then were embedded in liquid paraffin using the automated inclusion system EG: 1160 Leica. The blocks were sectioned (4 microns) and stained Hematoxylin and Eosin for histopathological studies. To investigate the occurrence of hyperplasia, immunohistochemistry was carried out using KI 67 (primary antibody diluted in PBS 1:100, Dako, Carpinteria, CA, USA), BA2000 and Pk6100 (secondary antibodies diluted in PBS 1:200, Dako, Carpinteria, CA, USA). 
For the different groups, the Mac Neman test was used in the study of the combination of urine culture and uninhibited contractions in the beginning and the end of the evaluation. Comparison of the groups' mean profile along both moments of evaluation was performed through the analysis of repeated measures, considering both groups independently. For the histological and immunohistochemical analysis, the Goodman test was used.

\section{RESULTS}

Four weeks after surgery, bladder weight in $\mathrm{G} 2$ was 2.5 times heavier than $\mathrm{G} 1(\mathrm{p}<0.05)$. Partial outlet obstruction was also responsible for an increase in bladder infection. Urine samples collected at M2 showed that $93 \%$ of $\mathrm{G} 2$ had positive urine cultures compared to $13 \%$ in the G1 group ( $\mathrm{p}<0.001$ ).

The cystometric studies showed that detrusor instability was observed in all G2 animals (Figure-1). In G2, maximum cystometric capacity (Volmax) was higher $(\mathrm{p}<0.05)$ at $\mathrm{M} 2$ if compared to M1 (Table-1). Maximum detrusor pressure and bladder compliance showed no statistical difference between M1 and M2 in both groups (Table-1).

The histopathological analysis showed that animals in G1 had normal epithelium at the end of the trial. However, animals in G2 underwent histological changes (Table-2), including muscle hypertrophy (94\%) (Figure-2), Inflammatory reaction (87\%), mild to moderate fibrosis of the adventitia (80\%) (Figure-3) and mild to moderate fibrosis of the mucosa (53\%), Figure- 4 .

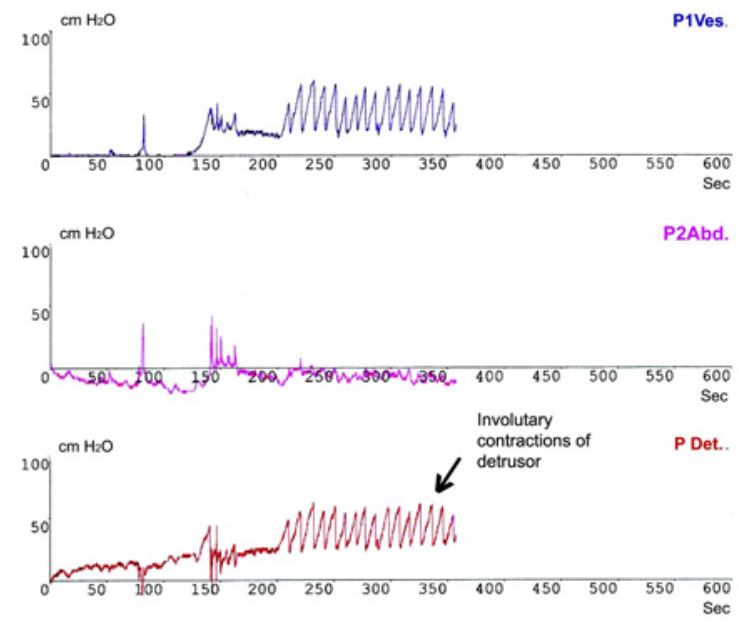

Figure 1 - Cystometric study of a rabbit in the group with partial bladder outlet obstruction (G2). A) Vesical pressure (P1Ves) in $\mathrm{cm} \mathrm{H}_{2} \mathrm{O}$. B) Abdominal pressure ( $\mathrm{P} 2 \mathrm{Abd}$ ) in $\mathrm{cm} \mathrm{H}_{2} \mathrm{O}$. C) Invol-

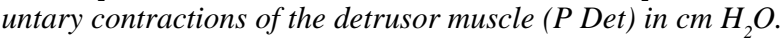

The immunohistochemical analysis showed significantly higher hyperplasia of the epithelium and muscle layer in G2 (Figure-5).

\section{COMMENTS}

Increase in bladder weight is predominant in vesical obstruction models $(9,10)$. In this experiment, after 4 weeks of PBOO, bladder weight in G2 animals was 2.5 times that of controls. The increase in bladder weight is explained in part by the hypertrophy of the detrusor muscle, found in $98 \%$ of the G2 animals. This is in line with previous studies using a

Table 1 - Maximum vesical volume $(m L)$, maximum detrusor pressure $\left(\mathrm{cm} \mathrm{H}_{2} \mathrm{O}\right)$, vesical compliance $\left(\mathrm{mL} / \mathrm{cm} \mathrm{H}_{2} \mathrm{O}\right)$ in rabbits. Mean and standard deviation of cystometric parameters in the beginning (M1) and 4 weeks after surgery (M2) in group G1 (control) and G2 (infravesical obstruction).

\begin{tabular}{lcccc}
\hline & \multicolumn{2}{c}{ G1 } & \multicolumn{2}{c}{ G2 } \\
& M1 (Initial) & M2 (Final) & M1 (Initial) & M2 (Final) \\
\hline Maximum vesical volume & $36.13 \pm 24.78$ & $42.33 \pm 21.01$ & $35.13 \pm 13.60^{*}$ & $54.53 \pm 22.77^{*}$ \\
Maximum detrusor pressure & $36.47 \pm 15.03$ & $27.27 \pm 13.47$ & $36.60 \pm 14.47$ & $31.40 \pm 15.42$ \\
Vesical compliance & $2.79 \pm 1.47$ & $3.40 \pm 1.71$ & $2.81 \pm 2.10$ & $2.83 \pm 1.67$ \\
\hline
\end{tabular}

$* p<0.05$ 
Table 2 - Percentage of adventitial, mucosal and muscle interstitial fibrosis, inflammatory reaction, muscle hypertrophy and epithelium ulceration observed in the group with partial bladder outlet obstruction (G2) 4 weeks after surgery.

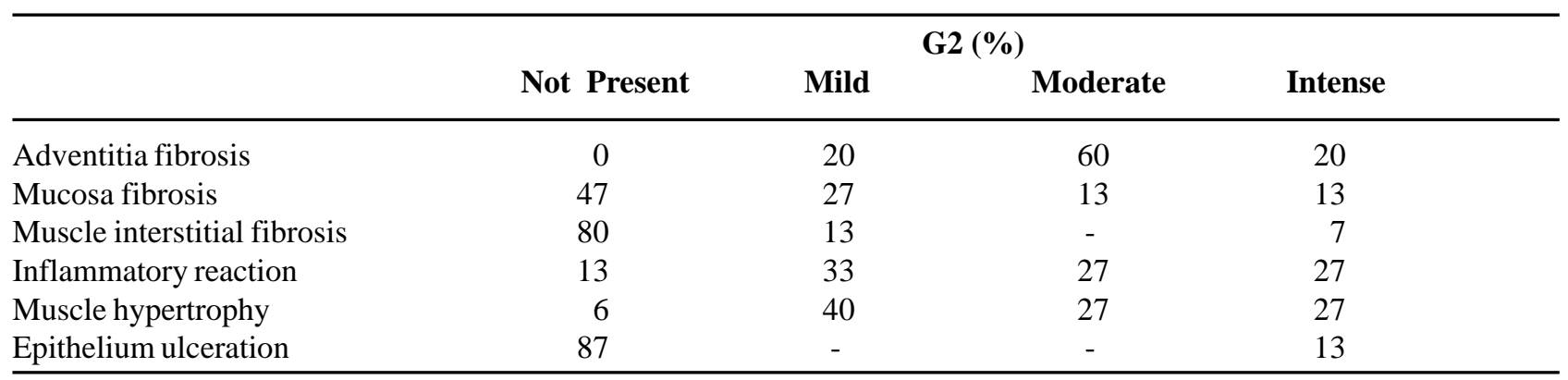

rabbit model of BOO (11). Indeed, the hypertrophy of smooth muscle (SM) cells in the bladder of obstructed rabbits, was followed by changes in the expression of cytoskeleton and cytocontractile proteins such as SM alpha-actin and SM myosin (12). Recent studies suggest that the mechanical stretch due to obstruction is responsible for stimulating the expression of growth factors and other specific proteins through the activation of stretch-activated ion channels (SACs) and protein kinase (PKC) sarcolemal proteins (13).

The immunohistochemistry analysis, using the cell proliferation marker KI 67, showed that hyperplasia of the epithelium and muscle cells was also responsible for the $\mathrm{G} 2$ group increase in bladder weight. This confirms earlier studies where hyperplasia of the extramural conjunctive tissue (14), epithelium and muscle cells $(15,16)$ was observed in animals with bladder obstruction.

A high incidence of positive urine cultures in G2 (93\%) after partial obstruction is most likely a combination of overdistesion of the bladder and animal manipulation. In G2 $87 \%$ of the bladder epithelium had severe inflammatory reaction and $13 \%$ presented ulcerations. At M2, the urine culture of G2 revealed a predominance of Escherichia coli (data not shown). This corroborates with earlier data which demonstrated that the integrity of the urothelium is compromised after the bladder is stretched $(17,18)$, thus making it easier for bacterial colonization.
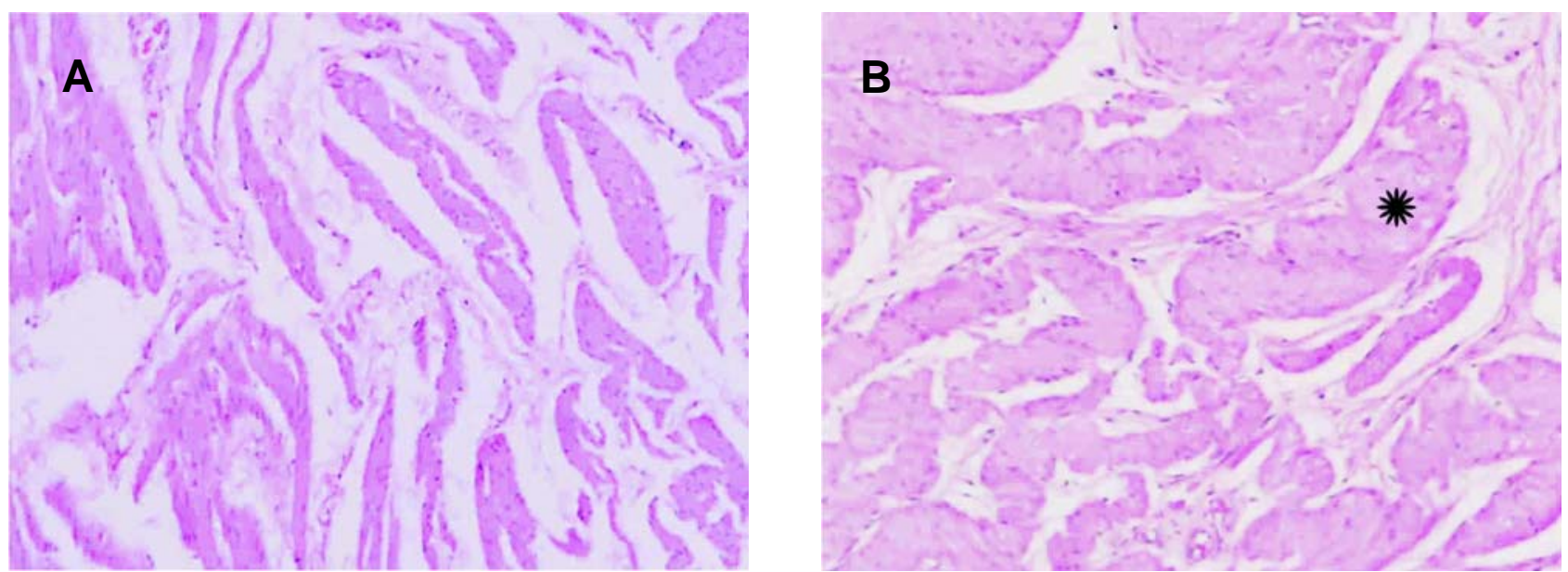

Figure 2 - Histological section of rabbit bladder. A) Absence of muscular hypertrophy in group G1 (HE, X40). B) Intense hypertrophy in group G2 (asterisk), HE X100. 

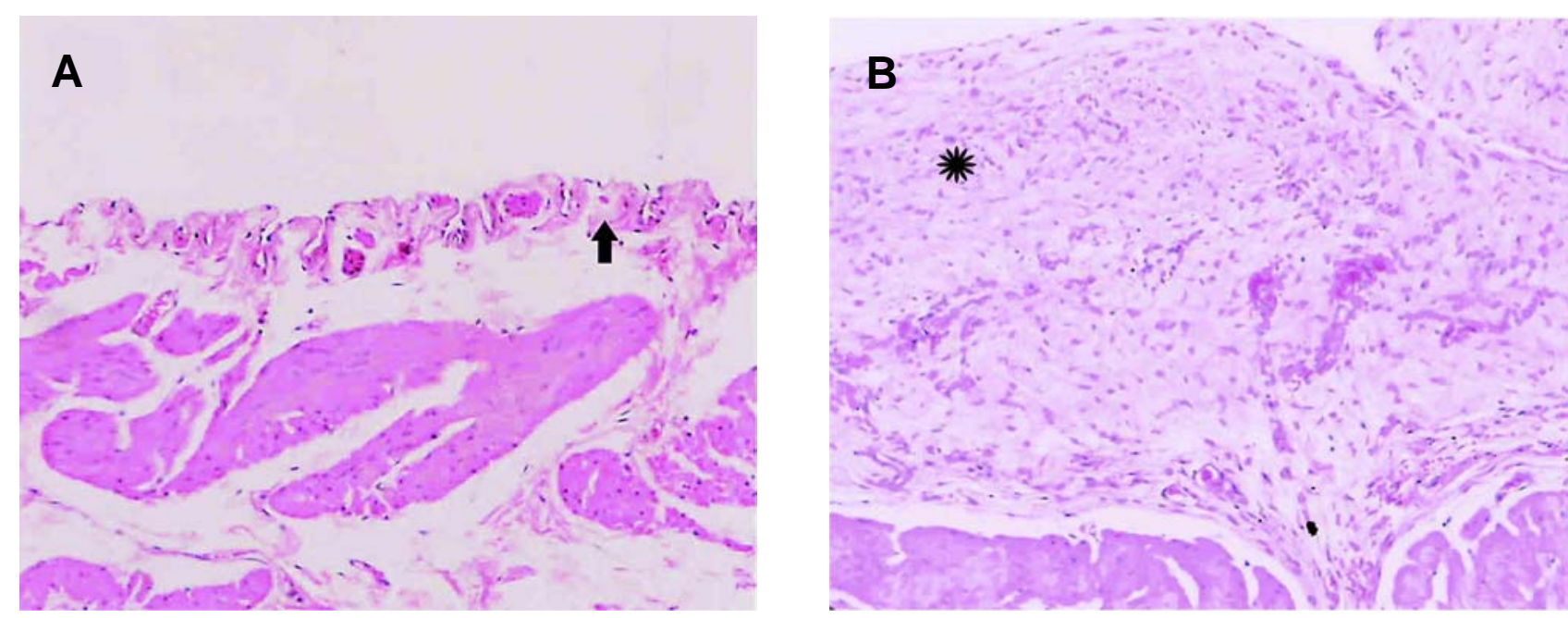

Figure 3 - Histological section of rabbit bladder. A) Absence of adventitial fibrosis in group G1 (arrow), HE X40. B) Intense adventitial fibrosis in group G2 (asterisk), HE X100.
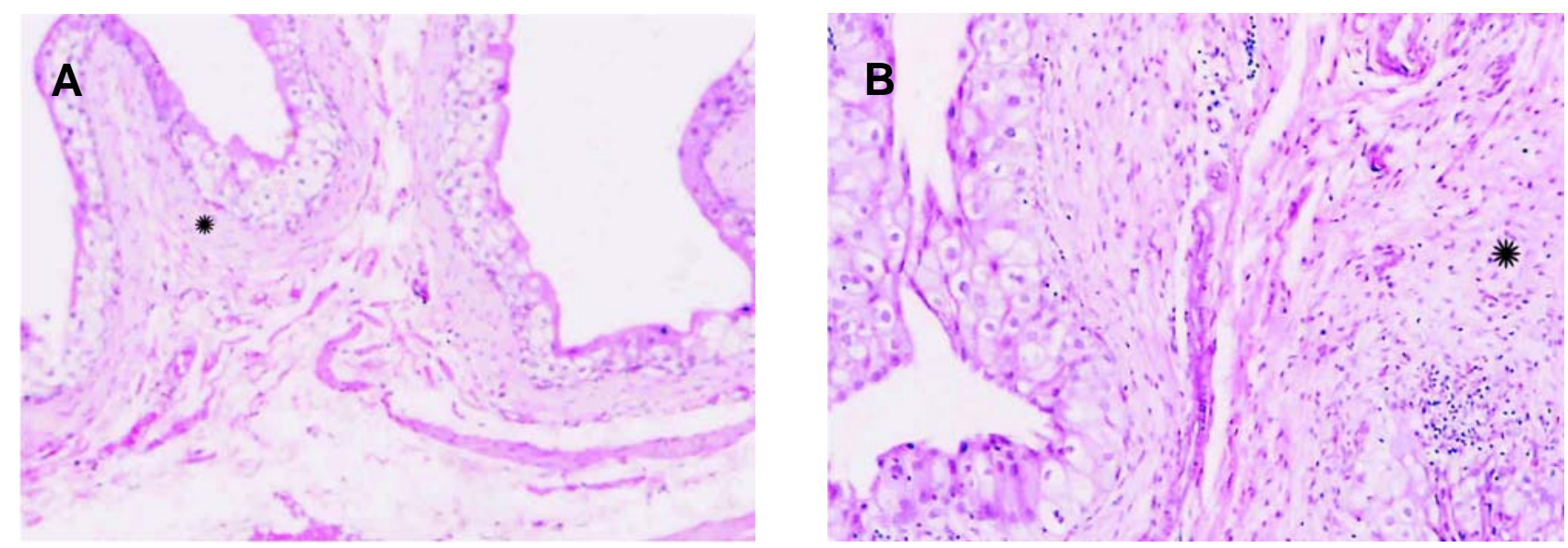

Figure 4 - Histological section of rabbit bladder. A) Absence of mucosa fibrosis in group G1 (asterisk), HE X100. B) Mild mucosa fibrosis (asterisk) in group G2, HE X100.

In the cystometric study, no significant difference was observed in maximum vesical volume (Volmax) at the different moments for G1. However, G2 had significantly $(\mathrm{p}<0.05)$ increased Volmax at M2 when compared to M1. In other partial obstruction models, using silk ligature $(19,20)$ and silicon sleeve (21) vesical capacity was also significantly increased.

There was no statistical difference in maximum detrusor pressure between groups at M1 and M2. These findings disagree with $(16,22)$, where an increase in detrusor pressure was observed, following BOO.
These discrepancies in the results are expected due to the different methodology used to cause BOO, the timing of the cystometric study (6) and individual differences (4). Recent experiments showed that despite detrusor hypertrophy, some bladders with BOO work normally while others are unable to empty properly (23). This was associated with over expression of nonmuscular caldesmon (l-CaD), a protein capable of inhibiting actin-activated myosin ATPase, compromising the detrusor contractions. The quantification of $1-$ $\mathrm{CaD}$ in further $\mathrm{BOO}$ studies could be useful for separating the different degrees of detrusor dysfunction. 

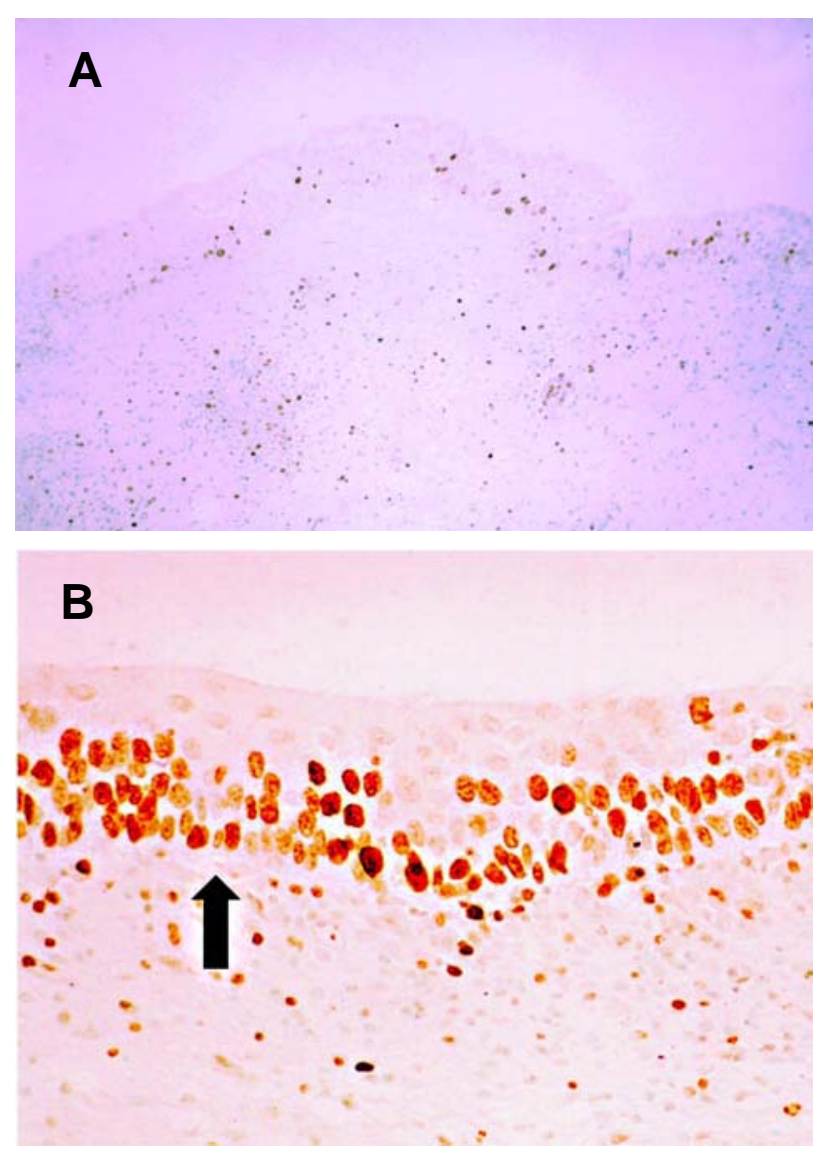

Figure 5 - Histological section of rabbit bladder immunohistochemical staining (KI 67 antibodies), X400. A) Control group - G1. B) Intense epithelial hyperplasia (arrow) in group G2, X400.

Lost of vesical compliance is associated with lost of elasticity due to increase in conjunctive tissue deposition in different layers of the bladder. Cystometric studies showed no difference in vesical compliance between groups. This is supported by histological data where mild to moderate fibrosis of the mucosa and adventitial layers were predominant and interstitial muscle fibrosis was absent in $80 \%$ of the G2 group; thus, not yet compromising vesical compliance. However, other studies showed higher incidence of mucosal (16), adventitial (15) and interstitial muscle (24) fibrosis, suggesting that the G2 group could develop loss in vesical compliance if $\mathrm{PBOO}$ had persisted for longer.
Detrusor instability was persistent in G2 at M2 while there was an absence of contraction in the control group. This demonstrates that the experimental model employed is adequate for studying vesical instability. This is extremely important for it will enable further studies on the role of muscarine receptors subtypes $(25,26)$, adrenoreceptors subtypes $(27,28)$, and myosin isoform (29) expression, which have been described as being important in the control of contractility of the detrusor muscle.

\section{CONCLUSION}

The described experimental PBOO model in rabbits induced detrusor instability and histological alteration, similar to changes caused by obstructive pathologies, and therefore is a useful tool for further physiological and pharmacological research.

\section{ACKNOWLEDGEMENT}

This work was supported by the São Paulo Foundation for Research Support - FAPESP.

\section{CONFLICT OF INTEREST}

None declared.

\section{REFERENCES}

1. Andersson KE: Current concepts in the treatment of disorders of micturition. Drugs. 1988; 35: 477-94.

2. Abrams P, Wein AJ: Introduction: Overactive bladder and its treatments. Urology. 2000; 55 (Suppl. 5A): 12.

3. Sibley GN: An experimental model of detrusor instability in the obstructed pig. Br J Urol. 1985; 57: 2928.

4. Lluel P, Duquenne C, Martin D: Experimental bladder instability following bladder outlet obstruction in the female rat. J Urol. 1998; 160: 2253-7.

5. Schroder A, Chichester P, Kogan BA, Longhurst PA, Lieb J, Das AK, et al.: Effect of chronic bladder outlet obstruction on blood flow of the rabbit bladder. J Urol. 2001; 165: 640-6.

6. Gosling JA, Kung LS, Dixon JS, Horan P, Whitbeck C, Levin RM: Correlation between the structure and 
function of the rabbit urinary bladder following partial outlet obstruction. J Urol. 2000; 163: 1349-56.

7. Balasteghin KT, Nardo AM, Amaro JL, Padovani CR: Experimental model of bladder instability in rabbits. Int Braz J Urol. 2003; 29: 62-7.

8. Amaro JL, Cury PR, Fabris VH, Trindade JC: Ampliação vesical utilizando dura-máter e pericárdio bovino. Estudo comparativo em coelhos. J Bras Urol. 1997; 23: 88-92.

9. Levin RM, High J, Wein AJ: The effect of short-term obstruction on urinary bladder function in the rabbit. $\mathrm{J}$ Urol. 1984; 132: 789-91.

10. Nigro DA, Haugaard N, Wein AJ, Levin RM: Metabolic basis for contractile dysfunction following chronic parcial bladder outlet obstruction in rabbits. Mol Biol Biochem. 1999; 200: 1-6.

11. Kato K, Monson FC, Longhurst PA, Wein AJ, Haugaard N, Levin RM: The functional effects of longterm outlet obstruction on the rabbit urinary bladder. $\mathbf{J}$ Urol. 1990; 143: 600-6.

12. Roelofs M, Wein AJ, Monson FC, Passerini-Glazel G, Koteliansky VE, Sartore S, et al.: Contractility and phenotype transitions in serosal thickening of obstructed rabbit bladder. J Appl Physiol. 1995; 78: 143241.

13. Yamaguchi O: Response of bladder smooth muscle cells to obstruction: signal transduction and the role of mechanosensors. Urology. 2004; 63 (Suppl 1): 11-6.

14. Levin RM, Wein AJ, Buttyan R, Monson FC, Longhurst PA: Update on bladder smooth-muscle physiology. World J Urol. 1994; 12: 226-32.

15. Ghoniem GM, Regnier CH, Biancani P, Johnson L, Susset JG: Effect of vesical outlet obstruction on detrusor contractility and passive properties in rabbits. $\mathrm{J}$ Urol. 1986; 135: 1284-9.

16. Kuo HC: Effects of mild bladder outlet obstruction on rabbit bladder structure and function. J Formos Med Assoc. 1995; 94: 555-61.

17. Leppilahti M, Kallioinen M, Tammela TL: Duration of increased mucosal permeability of the urinary bladder after acute overdistension: an experimental study in rats. Urol Res. 1999; 27: 272-6.

18. Buttyan R, Chen MW, Levin RM: Animal models of bladder outlet obstruction and molecular insight into the basis for the development of bladder dysfunction. Eur Urol. 1997; 32: 32-9.

19. Malmgren A, Sjogren C, Uvelius B, Mattiasson A, Andersson KE, Andersson PO: Cystometrical evaluation of bladder instability in rats with infravesical outflow obstruction. J Urol. 1987; 137: 1291-4.
20. Steers WD, De Groat WC: Effect of bladder outlet obstruction on micturition reflex pathways in the rat. $\mathrm{J}$ Urol. 1988; 140: 864-71.

21. Kato K, Wein AJ, Kitada S, Haugaard N, Levin RM: The functional effect of mild outlet obstruction on the rabbit urinary bladder. J Urol. 1988; 140: 880-4.

22. Gray M: Progressive changes in detrusor function with bladder outlet obstruction. J Urol. 1997; 158: 631-5.

23. Zhang EY, Stein R, Chang S, Zheng Y, Zderic SA, Wein AJ, et al.: Smooth muscle hypertrophy following partial bladder outlet obstruction is associated with overexpression of non-muscle caldesmon. Am J Pathol. 2004; 164: 601-12.

24. German K, Bedwani J, Davies J, Brading AF, Stephenson TP: Physiological and morphometric studies into the pathophysiology of detrusor hyperreflexia in neuropathic patients. J Urol. 1995; 153: 1678-83.

25. Hegde SS, Eglen RM: Muscarinic receptor subtypes modulating smooth muscle contractility in the urinary bladder. Life Sci. 1999; 64: 419-28.

26. Chapple CR, Yamanishi T, Chess-Williams R: Muscarinic receptor subtypes and management of the overactive bladder. Urology. 2002; 60 (Suppl. 1): 82-8; discussion 88-9.

27. Wanajo I, Tomiyama Y, Yamazaki Y, Kojima M, Shibata N: Pharmacological characterization of betaadrenoceptor subtypes mediating relaxation in porcine isolated ureteral smooth muscle. J Urol. 2004; 172: 1155-9.

28. Andersson KE: Storage and voiding symptoms: pathophysiologic aspects. Urology. 2003; 62 (Suppl 2): 3-10.

29. Austin JC, Chacko SK, DiSanto M, Canning DA, Zderic SA: A male murine model of partial bladder outlet obstruction reveals changes in detrusor morphology, contractility and Myosin isoform expression. $\mathrm{J}$ Urol. 2004; 172: 1524-8.

Received: April 26, 2005

Accepted after revision: August 08, 2005
Correspondence address:
Dr. João Luiz Amaro
Faculdade de Medicina de Botucatu
Dept de Urologia, Distrito de Rubião Júnior, s/n
18618-970, Botucatu, SP, Brazil
Telephone / Fax: + 5514 3811-6271
E-mail: jamaro@fmb.unesp.br 


\section{EDITORIAL COMMENT}

The authors present an interesting experimental model as well as a careful experimental design conferring credibility to the results obtained.

The authors have pointed out fibrosis of the adventitia and "mild to moderate fibrosis of the adventitia and mild to moderate fibrosis of the mucosa". Immunohistochemical analysis showed a high hyperplasia of the epithelium and muscle layer. However, all observations are expressed in subjective assessments. In a work in which methodological care with different parameters involved in the experiment are clear, we would expect the use of morphometric methods (stereologic) which application has showed to be efficient and the results much more reliable than the method where crosses or personal assessments are used. The results expressed after the use of this methodology can be easily and correctly interpreted and, thus, open the way to researches that shall certainly result in efficient therapeutic conducts as foreseen by the authors.

\section{Dr. Waldemar Silva Costa} Urogenital Research Unit State University of Rio de Janeiro Rio de Janeiro, Brazil E-mail: wscosta@gmail.com 